\title{
Twist Grain Boundary Phases Giving Developable Domain Textures as Those Exhibited by Columnar Phases
}

\author{
Anjuli Khandelwal ${ }^{1}$, Maneesha Yadav $^{1}$, and S. S. Bawa \\ ${ }^{1}$ Department of Physics, S.D. (PG) College, Muzaffarnagar, UP \\ ${ }^{2}$ Polymeric and Soft Material Section, National Physical Laboratory, \\ New Delhi, India
}

This paper reports experimental observation of TGBA, TGBC* phases in a mixture of Liquid Crystals. The phases exhibit developable domain textures as those observed for columnar systems. Under polarizing microscope they appear as cylindrical and cone-like domain texture. In such domains the smectic blocks, the grain boundaries and the layers of helical structure of TGB phase are coiled in a double twist manner around an axis. A peculiar texture is obtained in the intermediate region between the TGBA and Cholesteric phase while cooling the sample from isotropic phase. This phase is identified as 'Twist Grain Boundary phase of Hexatics' (TGBH) proposed theoretically by Kamien. The phase reappears as TGBA is heated. The Twist grain boundary phase of Hexatics consists of twisted $N+6$ regions separated by grain boundaries. Within each region the bond order twists with pitch $2 \pi / q$ and the director is aligned in a nematic state. The director is rotated by some finite angle across a grain boundary moving along the pitch axis that is perpendicular to the nematic director.

Keywords: bond order; columnar phase; developable domains; liquid crystals; TGB

\section{INTRODUCTION}

Twist Grain Boundary Phases (TGB) have attracted a lot of attention in recent years. From different studies it has become clear that these phases are helical stack of smectic blocks. Any two-smectic blocks are connected through a twist grain boundary. The twist grain boundary is a periodic array of screw dislocations. The TGB phases are classified as commensurate or incommensurate depending on whether or not the

Anjuli Khandelwal wants to thank Prof. Ruben Garcia for funding her visit to this conference.

Address correspondence to Anjuli Khandelwal, Department of Physics, S.D. (PG) College, Muzaffarnagar 251001, UP. E-mail: anjuli.khandelwal@gmail.com 
net director rotation across the grain is a rational multiple of $2 \pi$. In the case of commensurate TGB phases the twist axis is an $\mathrm{N}$-fold screw axis where $\mathrm{N}$ is an integer. The in-commensurate structure has no such screw symmetry. Further the smectic blocks could be of smectic A ( $\mathrm{Sm} \mathrm{A})$, smectic C (Sm C) or smectic $\mathrm{C}^{*}\left(\mathrm{Sm} \mathrm{C} \mathrm{C}^{*}\right)$ structure. The TGB phases are then designated as TGBA, TGBC, TGBC* respectively.

The history of TGB phases started from 1972, when de Gennes [1] pointed out a strong analogy between the nematic $(\mathrm{N})$ to smectic $\mathrm{A}$ (Sm A) transition in liquid crystals and the normal to superconductor transition in metals. The possible existence of a liquid crystalline phase similar to the Abrikosov phases in type II superconductors led Renn and Lubensky (RL) [2] to predict the existence of frustrated SmA phase (TGBA phase) to be observed with chiral molecules of LCs between $\mathrm{N}^{*}$ and $\mathrm{SmA}$ phase. In the vicinity of a $\mathrm{N}^{*}-\mathrm{SmA}-\mathrm{SmC}^{*}$ point, Renn [3] also predicted the existence of TGBC and TGBC* phases. Phase diagrams involving all three TGB phases were calculated in the same paper.

The identification of TGB phases requires complicated X-rays, optical, calorimetric experiments. The TGBA phase was first characterized experimentally by Goodby et al. [4]. All the detail of a TGBA mesophase were experimentally observed and confirmed by Ihn et al. [5] using freeze fracture electron micrographs. The TGBC phase was discovered by Nguyen et al. [6]. However, the TGBC structure proposed by them was found to differ from the picture originally proposed by RL [2]. The existence of both commensurate and incommensurate TGB phases was confirmed by Navailles et al. [7].

Rebeiro et al. [8] have performed optical investigations on TGBA and TGBC phases. Kleman et al. [9] gave a theoretical approach to defects in a TGBA phase. Brunet et al. [10] proposed new TGBC structures based on splayed polarization twisted director structures. Pramod et al. [11] reported the discovery of a new twist grain boundary phase in binary mixture called the undulated TGBC* ${ }^{*}$ U-TGBC*): characterized by the appearance of translation symmetry-breaking by square grid pattern in the plane normal to the helix axis. Rebeiro et al. $[12,13]$ published results obtained with a single component liquid crystalline material. By optical and X ray studies, they showed that the TGBC phase (S-TGBC) exhibits a square grid pattern in planner geometry and a very board $\omega$ scan profile of layer normal directions relative to the TGB helix axis. Dhar [14] and Pandey et al. [15] have reported TGB phases in mixtures of Liquid Crystals. We report TGBA and TGBC $^{*}$ phases giving cylindrical and cone-like domain textures (CC type textures) in mixtures of liquid crystals [16]. 
TGB phases are more closely related to high $\mathrm{T}_{\mathrm{c}}$ superconductors that are essentially type II and unlike low $\mathrm{T}_{\mathrm{c}}$ superconductors, their critical domain is broad and the effect of fluctuations is important. In type II case, thermal fluctuations can cause the Abrikosov vortex lattice to melt, hence producing an entangled flux liquid in which the flux lines are on average parallel to a common direction $[17,18]$. In a similar fashion the TGB phase could also melt to a chiral line liquid just as the abrikosov phase melts to a directed line liquid. Kamien and Lubensky [19] have proposed a model of directed lines to describe the liquid of screw dislocations in the TGB phases in which the screw dislocations exhibit no long range positional order, but are arranged in a helical fashion to form a cholesteric LC. This phase is given designation $\mathrm{N}_{\mathrm{L}}{ }^{*}$. Navilles et al. [20] performed high-resolution calorimetric studies to investigate the phase diagram of several LC series with a SmA-TGBA-Cholesteric sequence. The calorimetric data are consistent with the existence of the $\mathrm{N}_{\mathrm{L}}{ }^{*}$ phase in an intermediate region between the TGBA and Cholesteric phase. Further, Navailles et al. [21] found with Synchrotron studies that the organisation of TGBA phase as a regular stack of well defined SmA slabs was lost in the intermediate region although the local SmA order was still strong.

On the other side Toner [22] proposed that nematic liquid crystals upon cooling could form a liquid crystalline phase with nematic order and Hexatic order in the plane perpendicular to it. When chiral molecules participate in $\mathrm{N}+6$ order, two kinds of twisting are allowed, Twisting of the nematic order, leading to the cholesteric states and twisting of the bond order, leading to braided Moire state [23]. The twisting of the bond order is analogous to the layered order of smectics with the pitch of the bond order equivalent to the layer spacing of the smectic. On the basis of these facts Kamien [24] proposed a new twist grain boundary phase of Hexatics (TGBH).

In the present paper results of careful polarized optical microscopy observations are performed on a mixture of Liquid Crystals.

\section{EXPERIMENTAL}

In this work, the results of systematic optical microscopic observations performed on a mixture of liquid crystals are presented. A mixture of Cholesteryl Nonanoate (CN) and p-ethoxy benzylidene-p-n-butyl aniline (EBBA) [7:3 by weight] was prepared. The mixing was done thoroughly in the isotropic state for one hour in vacuum. In this way well mixed samples with reproducible transition temperatures were obtained. 
Pure sample of Cholesteric Nonanoate $(\mathrm{CN})$ was procured from the New Jersey, USA, whereas N-(4-Ethoxy benzylidine)-4-butyle aniline (EBBA) was procured from Aldrich chemical company.

Binary mixture of CN and EBBA were prepared by weighing out pure samples using electro-balance of Precisa (Model ACS-205) having an accuracy $\pm 0.1 \mathrm{mg}$. A transmitted light polarizing microscope, OLYMPUS BX 51P, was used to identify the textures of different mesophases. Different mesophase transition temperatures were determined by using a Differential Scanning Calorimeter (DSC) of Mettler Toledo (Model DSC822 with STAR software) at $5.0^{\circ} \mathrm{C} / \mathrm{min}$ scanning rate.

\section{RESULTS AND DISCUSSION}

The DSC thermo gram recorded at $5{ }^{\circ} \mathrm{C} /$ min scanning rate (Fig. 1) confirms the existence of various phases recorded by optical microscopy. The temperatures recorded in the DSC thermo gram are different as compared to optical microscopic results. This may be because of the fact that in optical microscopic observations the sample is not perfectly isolated from the surroundings.

The polarizing microscopic pictures show new TGBH phase (Fig. 2) at $55^{\circ} \mathrm{C}$. In this texture along with cylindrical and cone like domain texture (CC type texture- similar to that observed for columnar phases), one can clearly see several radial streaks originating from the eyes of the CC domains. The transition to TGBA phase is marked by a color change (Fig. 3 ) at $45^{\circ} \mathrm{C}$. Figure 4 shows TGBA phase at $42^{\circ} \mathrm{C}$.

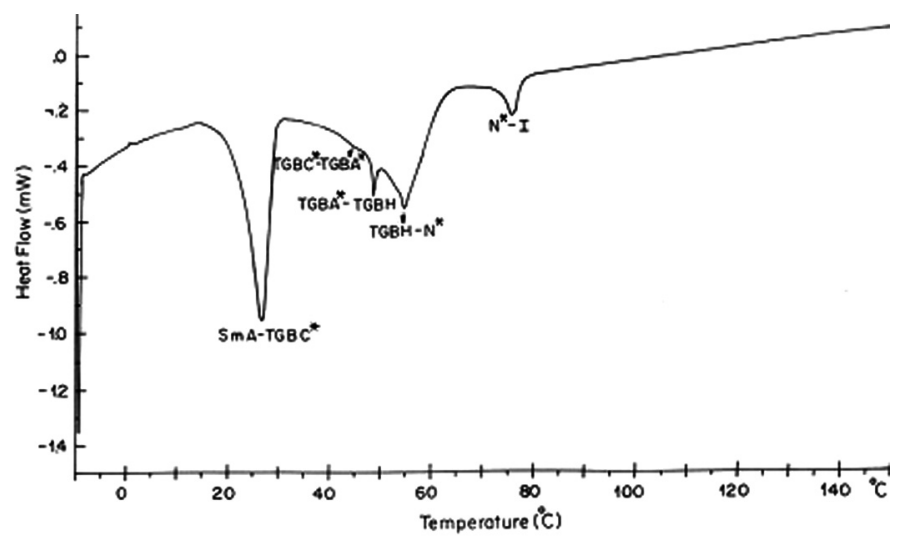

FIGURE 1 DSC thermogram recorded at $5^{\circ} \mathrm{C} / \mathrm{min}$ scanning rate. 


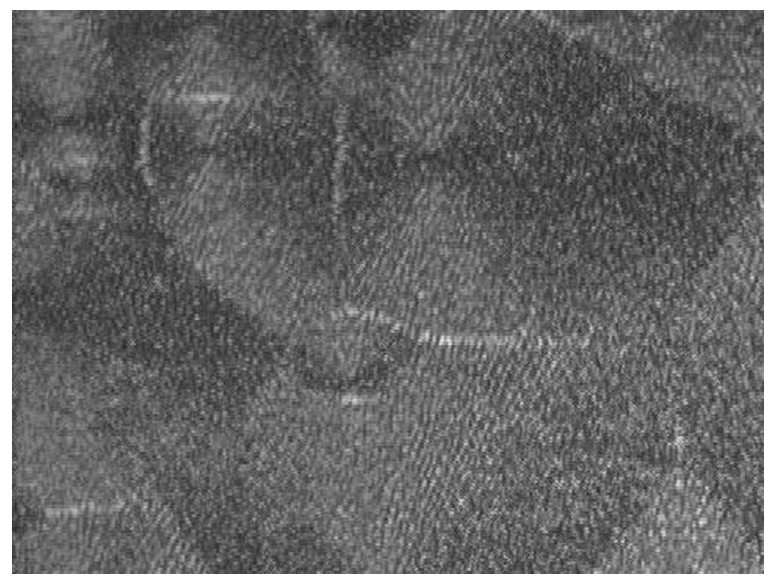

FIGURE 2 CC type texture with radial streaks originating from the eyes of the CC domains in TGBH phase $(\times 100), 55^{\circ} \mathrm{C}$.

The CC type texture (Fig. 4) at $42{ }^{\circ} \mathrm{C}$ is similar to the textures recorded by Ribeiro et al. [8] and by us [16] for TGBA phases. Figure 5 shows TGBA phase just before the transformation to TGBC* Figure 6 shows perfect $\mathrm{TGBC}^{*}$ phase at $36^{\circ} \mathrm{C}$. Here the $\mathrm{CC}$ domains are decorated with fracture lines. These lines appear very faint first and get more and more visible on decreasing temperature. They seem to approach a point as the temperature is lowered. This point seems to correspond

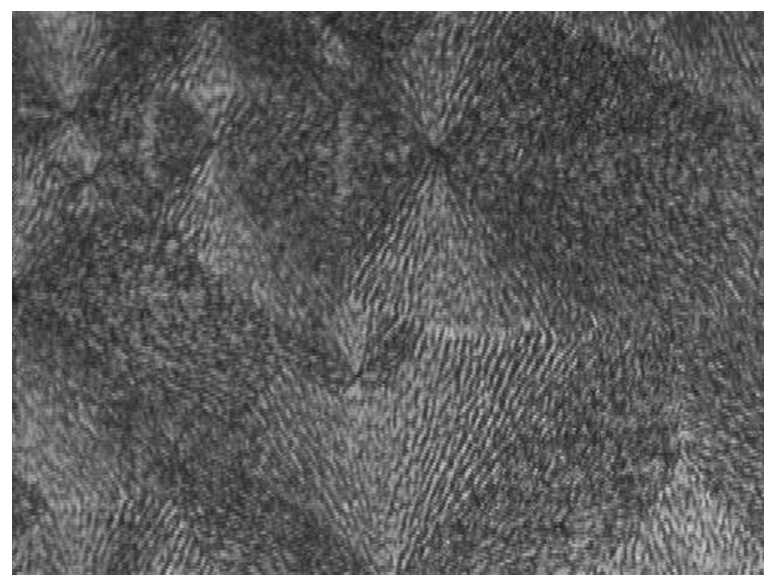

FIGURE 3 TGBH phase just before the transition to TGBA phase $(\times 100)$, $45^{\circ} \mathrm{C}$. 


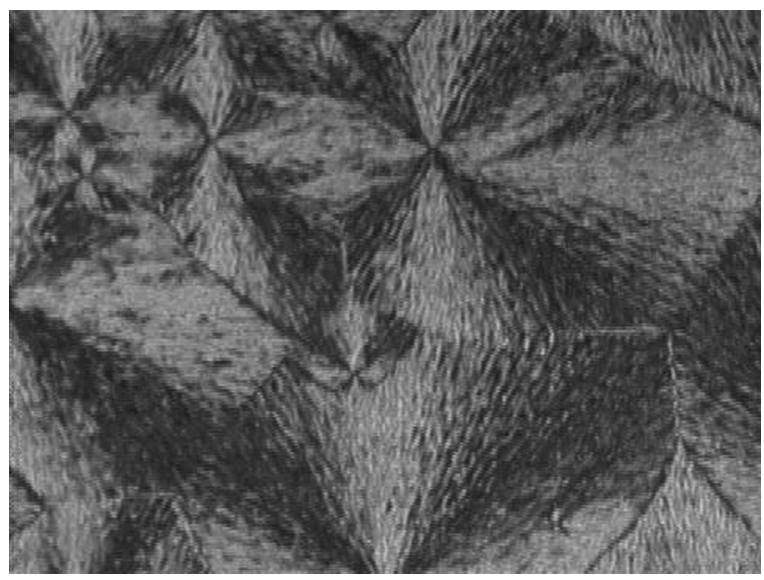

FIGURE 4 TGBH-TGBA transition $(\times 100), 42^{\circ} \mathrm{C}$.

to a $\chi$-line (edge dislocation line in the cholesteric ordering, or unwinding line) roughly perpendicular to the preparation. It appears that the helix is unwinding as the temperature is decreased (Fig. 7).

Figure 8 shows TGBH phase in another preparation of same sample in a $6 \mu$ thick sample when the sample is cooled from the isotropic phase via the cholesteric phase. Figure 9 shows TGBH-TGBA transition. Perfect TGBA phase is shown in Figure 10. There is change

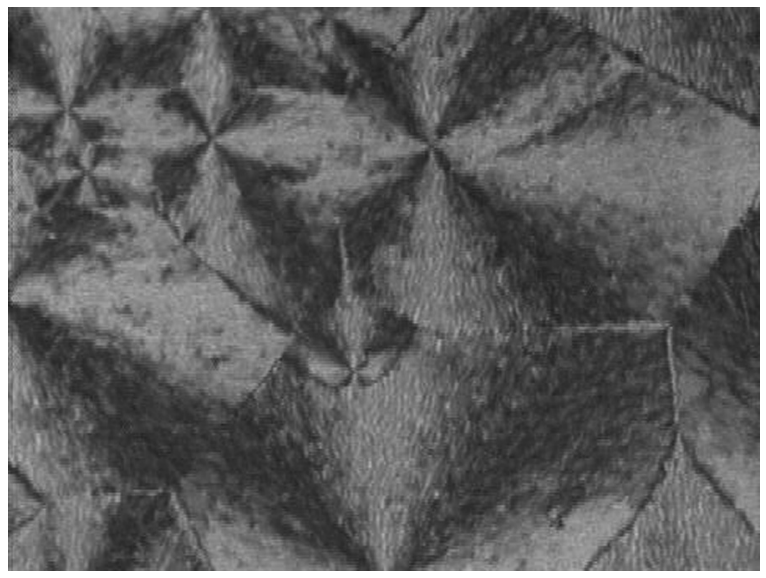

FIGURE 5 Well grown CC type domains in TGBA phase $(\times 100), 42^{\circ} \mathrm{C}$. 


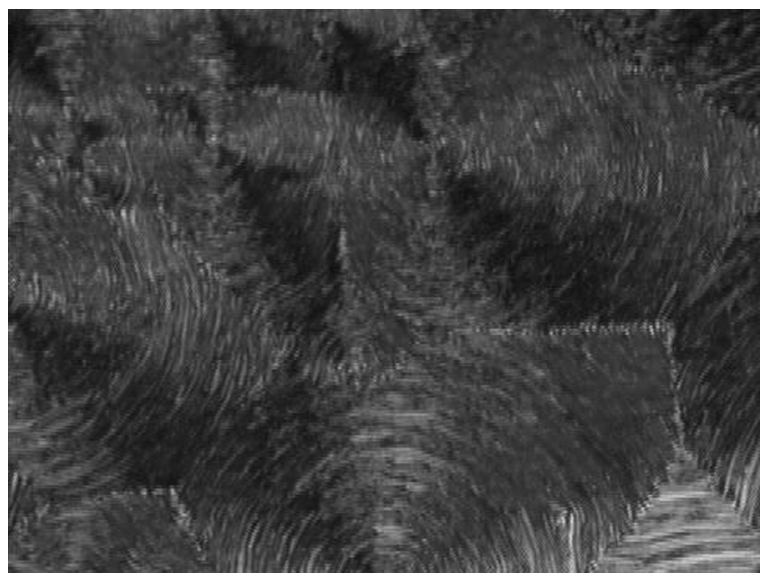

FIGURE 6 TGBC $^{*}$ phase with fracture lines, $(\times 100), 36^{\circ} \mathrm{C}$.

of transmission colour from violet to blue on cooling this phase. Further cooling gives usual Sm A phase.

On heating TGBA phase TGBH phase reappears. TGBH phase on heating gives way to planar texture of cholesteric (Ch) phase. Figures 11 to 15 show successively TGBA-TGBH, TGBH phase, TGBH-Ch phase transition, Ch phase and Ch-I transition.

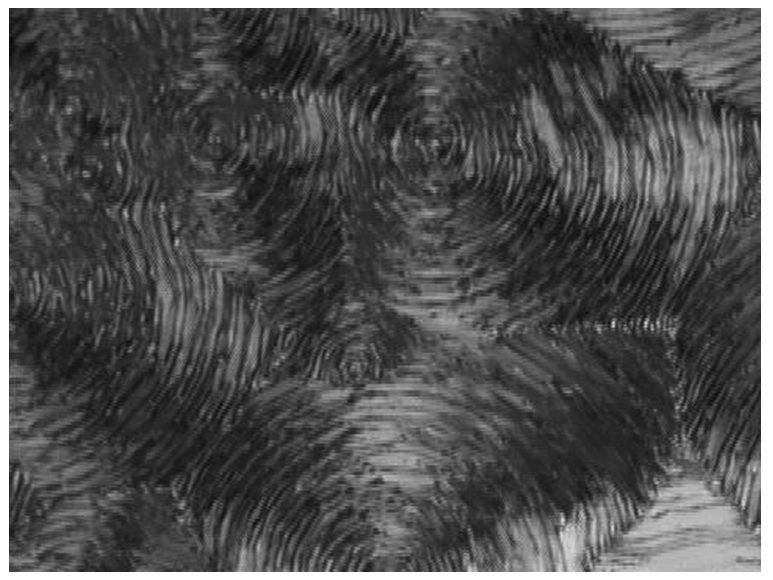

FIGURE 7 TGBC* phase at a lower temperature, $(\times 100), 34^{\circ} \mathrm{C}$. 


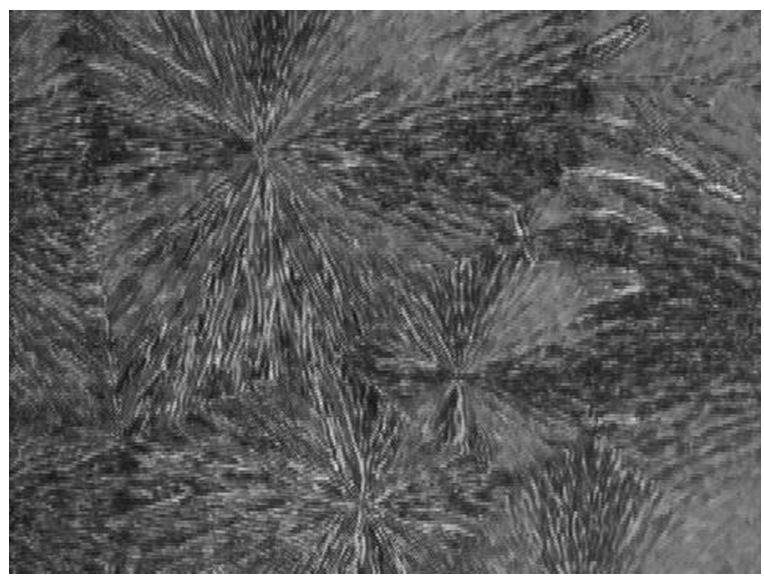

FIGURE 8 TGBH phase in a $6 \mu$ sample $(\times 100), 45^{\circ} \mathrm{C}$.

\section{CONCLUSION}

It is a well-known fact now that TGB phase, which is analog of the flux phase of superconductors, consists of a twisted lattice of screw dislocations as a counterpart of the hexagonal lattice of flux lines. In this frustrated Smectic phase, there are blocks of Smectic layers arranged in a helical way along an axis parallel to the smectic layers. Two adjacent Smectic blocks are rotated by an angle and they are separated by a grain boundary where a lattice of parallel and equidistant screw

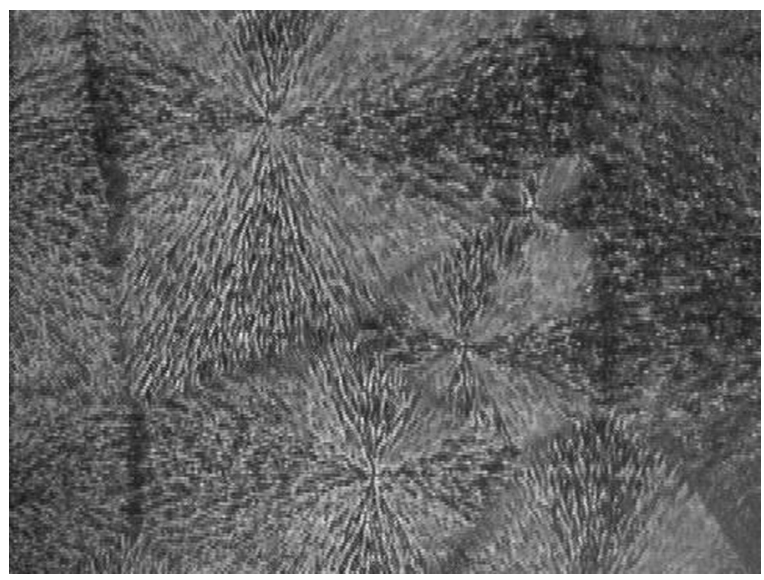

FIGURE 9 TGBH-TGBA transition in a $6 \mu$ thick sample $(\times 100), 30^{\circ} \mathrm{C}$. 


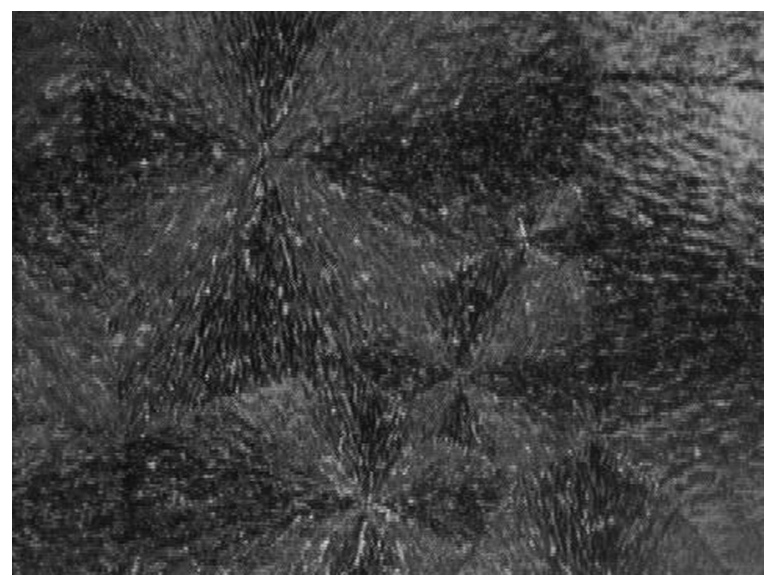

FIGURE 10 TGBA phase in a $6 \mu$ sample $(\times 100), 22^{\circ} \mathrm{C}$.

dislocation lines exists. Thus between two adjacent slabs, which mark the place where smectic layers go continuously from one slab to the other, a kind of twisted ribbon of molecules is formed.

In this structure two-dimensional columnar ordering exists only in the grain boundaries. Within the slabs, the columns are interrupted and merge back in to smectic layers. Never the less, there lateral dimensions are fixed, they have a constant density and it is possible to track them from a grain boundary to the next one. This ensures

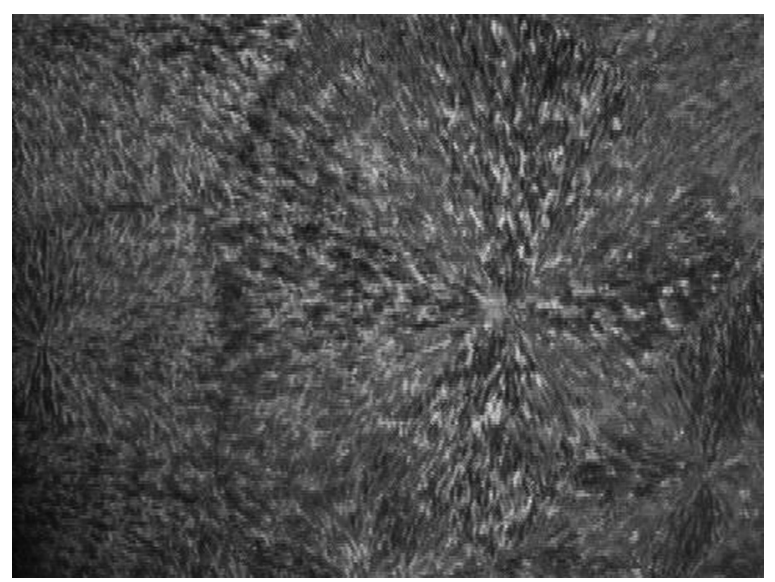

FIGURE 11 Reappearance of TGBH phase on heating TGBA, $33^{\circ} \mathrm{C}$. 


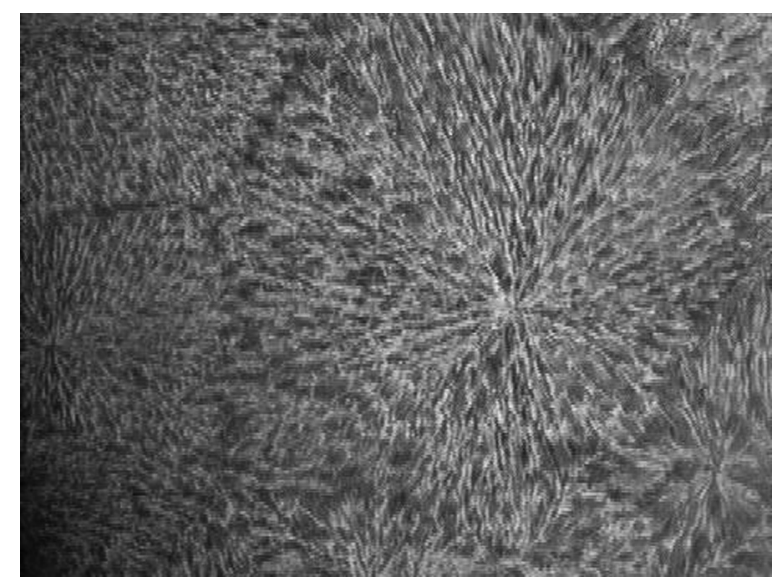

FIGURE $12 \mathrm{TGBH}$ as obtained from TGBA phase, $36^{\circ} \mathrm{C}$.

the formation of developable domains as in the classical columnar structures made of continuous columns [25]. Under polarizing microscope these phases appear as Cylindrical and cone-like domains (CC-type Texture). In such domains the smectic blocks, the grain boundaries and the layers of helical structure of TGB phase are coiled in a double twist manner around an axis [12]. The TGBA phase recorded in the present work shows well-formed CC-type textures similar to those recorded for TGBA phase $[8,12,15,16]$. But the phase

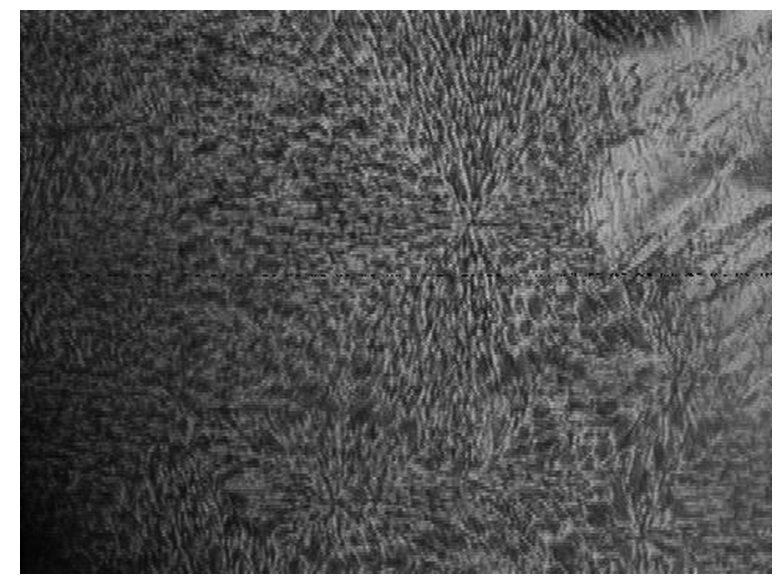

FIGURE 13 TGBH-cholesteric transition, $60^{\circ} \mathrm{C}$. 


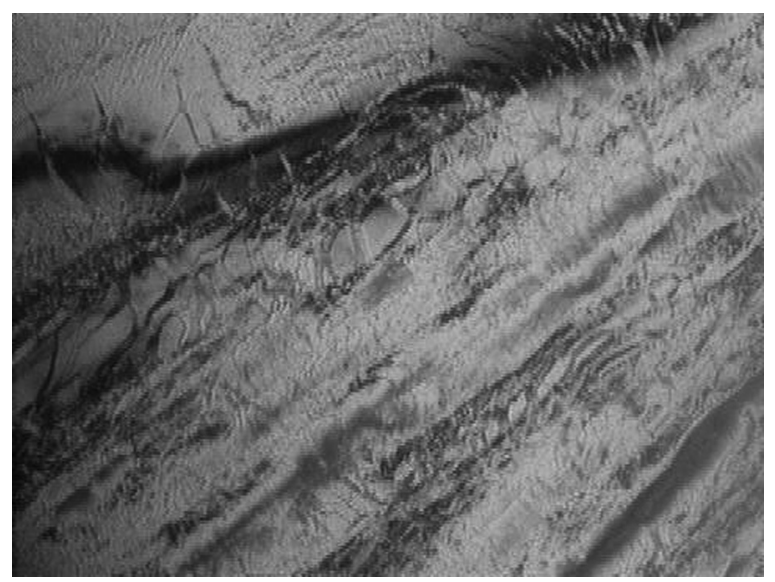

FIGURE 14 Cholesteric texture obtained on heating TGBH phase, $60^{\circ} \mathrm{C}$.

recorded between the cholesteric and TGBA region is different and appears to have some sort of hexatic order.

This twist grain boundary phase of Hexatics (TGBH) was proposed theoretically by Kamien [24]. We believe that the textures recorded in the present case belong to this three dimensional Defect-riddled phase.

In the sample studied, the phase sequence starts with cholesteric at high temperature. As the temperature is lowered, the sample forms TGBH phase. Recalling the phenomenology of the superconductors

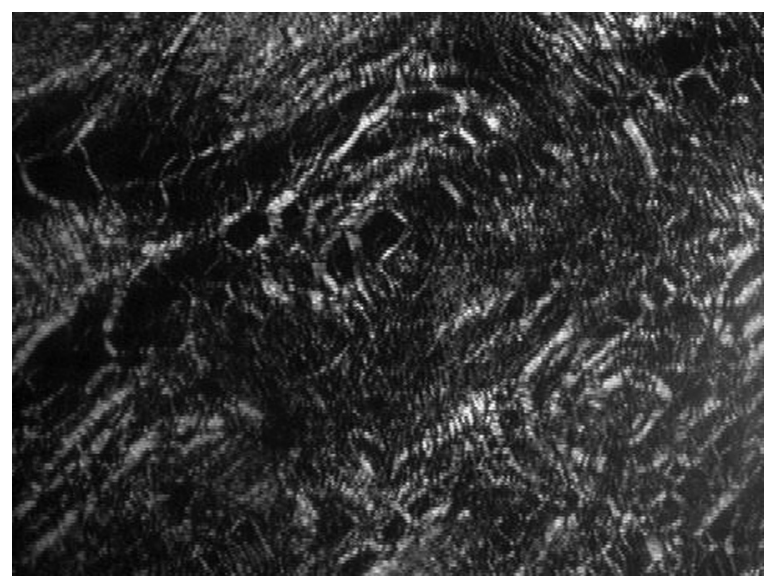

FIGURE 15 Planar cholesteric texture below $\mathrm{Ch}-\mathrm{I}$ transition, $69^{\circ} \mathrm{C}$. 
or smectic A liquid crystals, in the transformation $\mathrm{Ch}$ to smectic $\mathrm{A}$, the cholesteric twist is excluded in favour of chiral hexatic order [17].

This TGBH consists of regions of twisted $\mathrm{N}+6$ regions separated by grain boundaries made up of Hexatic disclinations. Within each region the bond order will twist with pitch $2 \pi / q$ and the director will be well aligned in a nematic state. As one moves along a pitch axis perpendicular to the nematic director, across a grain boundary, the nematic direction will jump by some finite angle $\alpha$. This will lead to a state which, at long distances, appear to be pure cholesteric, but will in fact have regions with rotating hexatic order [24].

\section{REFERENCES}

[1] de Gennes, P. G. (1972). Solid State Commun., 10, 753.

[2] Renn, S. R. \& Lubensky, T. (1988). Phys. Rev. A, 38, 2132.

[3] Renn, S. R. (1992). Phys. Rev. A, 45, 953.

[4] Goodby, J. W., Waugh, M. A., Stein, S. M., Chin, E., Pindak, R., \& Patel, J. S. (1989). Nature, 337, 449; J. Am. Chem. Soc., 111, 8119.

[5] Ihn, K. J., Zasadzinski, J. A., Pindak, R., Slaney, A. J., \& Goodby, J. W. (1992). Science, 258, 275.

[6] Nguyen, H. T., Bouchta, A., Navailles, L., Barois, P., Isaert, N., Twieg, R. J., Maaroufi, A., \& Destrade, C. (1992). J. Phys. II (France), 2, 1889.

[7] Navailles, L., Pindak, R., Barois, P., \& Nguyen, H. T. (1995). Phys. Rev. Lett., 74, 5224.

[8] Ribeiro, A. C., Nguyen, H. T., Galerne, Y., \& Guillon, D. (2000). Liquid Crystals, $27,27$.

[9] Kleman, M., Nastishin.Yu. A., \& Malthete, J. (2002). Eur. Phys. J. E., 8, 67.

[10] Brunet, M., Navailles, L., \& Clark, N. A. (2002). Eur. Phys. J. E., 7, 5.

[11] Pramod, P. A., Pratibha, R., \& Madhusudhana, N. (1997). Current Sci., 73, 761.

[12] Ribeiro, A. C., Oswald, L., Niscoud, J. F., Guillon, D., \& Galerne, Y. (1998). Eur. Phys. J. B., 1, 503.

[13] Ribeiro, A. C., Barois, P., Galerne, Y., Oswald, L., \& Guillon, D. (1999). Eur. Phys. J. $B, 11,121$.

[14] Dhar, R. (2006). Phase Transitions, 79(3), 175.

[15] Pandey, M. B., Dhar, R., Achalkumar, A. S., \& Yelamaggad, C. V. (2007). J. Phys. Condens. Matter, 19, 436219.

[16] Khandelwal, A., Bawa, S. S., \& Yadav, M. 'Optical and thermodynamical investigations of Twist Grain Boundary Phases in Liquid Crystals' simultaneously submitted to 'Phase Transitions'.

[17] Nelson, D. (1988). Phys. Rev. Lett., 60, 1973.

[18] Nelson, D. \& Seung, H. (1989). Phy. Rev. B, 39, 9153.

[19] Kamien, R. D. \& Lubensky, T. (1993). J. Phys. I, 3, 2131.

[20] Navilles, L., Garland, C. W., \& Nguyen, H. T. (1996). J. Phys. II (France), 6, 1243.

[21] Navailles, L. (1998). Phy. Rev. Lett., 81(19), 4168.

[22] Toner, J. (1983). Phy. Rev. A, 27, 1157.

[23] Kamien, R. D. \& Nelson, D. R. (1995). Phy. Rev. Lett., 74, 2499.

[24] Kamien, R. D. (1997). J. Phys. II (France), 6,461 (1996), 7,743.

[25] Ribiero, A. C., Dreyer, A., Oswald, L., Nicoud, J. F., Soldera, A., Guillon, D., \& Galerne, Y. (1994). J. Phys. II France, 4, 407. 